\title{
Análises físico-químicas e microbiológicas da água mineral comercializada em galões de 20 litros na Cidade de Belém-Pará
}

Physicochemical and microbiological analyzes of mineral water sold in 20 liter gallons in the City of Belém-Pará

Análisis fisico-químicos y microbiológicos de agua mineral vendida en galones de 20 litros en la Ciudad de Belém-Pará

\section{Resumo}

As atividades antrópicas podem afetar a qualidade da água mineral, favorecendo a veiculação hídrica de doenças. Nesse sentido, o presente trabalho teve como objetivo avaliar a qualidade microbiológica e físico-química das águas minerais comercializadas em Belém - Pará em galões de 20 litros, cujas análises foram realizadas no LACEN-PA no período de 01/01/2020 a 01/03/2021. Foram coletadas 15 amostras de diferentes marcas envasadas em galões de 20 litros e analisados os parâmetros físico-químicos e microbiológicos, conforme recomendações da RDC $\mathrm{n}^{\circ} 275$ de 22 de 2005 e da RDC $n^{\circ} 274$ de 2005. Os resultados microbiológicos revelaram que das 15 amostras analisadas 3 estavam impróprias para o consumo, pois foi detectada a presença de Coliformes totais e Clostrídios em 1 amostra e em 2 amostras apenas Coliformes totais. Já os parâmetros físico-químicos todas as amostras estavam de acordo com a determinação legal nos parâmetros analisados, sendo que as amostras apresentaram o pH entre 4,28 e 5,08 de acordo com o declarado no rótulo. Dessa forma, foi observado que a maioria das amostras analisadas estavam de acordo com padrões estabelecidos, exceto três amostras que apresentaram contaminação de microorganismos. Porém, é necessário um maior rigor em todas as etapas do controle de qualidade, bem como nas redes de distribuição, a fim de que a qualidade da água produzida seja garantida até o consumidor final.

Palavras-chave: Água mineral; Análises microbiológica; Análises físico-química; Saúde pública.

\begin{abstract}
Anthropogenic activities can affect the quality of mineral water, favoring the waterborne transmission of diseases. In this sense, this study aimed to evaluate the microbiological and physical-chemical quality of mineral waters sold in Belém - Pará in 20 liters, whose analyzes were carried out at LACEN-PA from 01/01/2020 to 01/ 03/2021. Fifteen samples of different brands were collected and filled in 20 liter cans and analyzed the physical-chemical and microbiological parameters, as recommended by RDC No. 275 of 22 of 2005 and RDC No. 274 of 2005 . The microbiological results revealed that of the 15 samples 3 analyzed were unfit for consumption, as the presence of total Coliforms and Clostridia was detected in 1 sample and in 2 samples only total Coliforms. As for the physicochemical parameters, all samples were in accordance with the legal determination in the analyzed parameters, and the samples had a pH between 4.28 and 5.08, as stated on the label. Thus, it was observed that most of the samples analyzed were in accordance with established standards, except for three samples that showed contamination by microorganisms.
\end{abstract}


However, greater rigor is needed at all stages of quality control, as well as in the distribution networks, so that the quality of the water produced is guaranteed to the final consumer.

Keywords: Mineral water; Microbiological analysis; Physicochemical analysis; Public health.

\section{Resumen}

Las actividades antropogénicas pueden afectar la calidad del agua mineral, favoreciendo la transmisión de enfermedades a través del agua. En este sentido, este estudio tuvo como objetivo evaluar la calidad microbiológica y físico-química de las aguas minerales comercializadas en Belém - Pará en 20 litros, cuyos análisis se realizaron en LACEN-PA del 01/01/2020 al 01/03/2021. Se recolectaron quince muestras de diferentes marcas y se llenaron en botes de 20 litros y se analizaron los parámetros físico-químicos y microbiológicos, según lo recomendado por el RDC No. 275 de 22 de 2005 y el RDC No. 274 de 2005. Los resultados microbiológicos revelaron que de los 15 las 3 muestras analizadas no eran aptas para el consumo, ya que se detectó la presencia de Coliformes Totales y Clostridios en 1 muestra y en 2 muestras solo Coliformes totales. En cuanto a los parámetros fisicoquímicos, todas las muestras estuvieron de acuerdo con la determinación legal en los parámetros analizados, y las muestras tuvieron un pH entre 4.28 y 5.08 , como se indica en la etiqueta. Así, se observó que la mayoría de las muestras analizadas cumplieron con los estándares establecidos, a excepción de tres muestras que presentaron contaminación por microorganismos. Sin embargo, se necesita un mayor rigor en todas las etapas del control de calidad, así como en las redes de distribución, para que la calidad del agua producida esté garantizada al consumidor final.

Palabras clave: Agua mineral; Análisis microbiológico; Análisis físico-químico; Salud pública.

\section{Introdução}

A água é um dos recursos naturais mais importantes no planeta e até bem pouco tempo era considerado como um bem infinito. $\mathrm{O}$ aumento da população e fatores como o crescimento econômico/industrial está causando degradação dos recursos hídricos por causa de seus múltiplos usos, destacando-se entre a agricultura, o abastecimento público, a pecuária, a indústria, a geração de energia, o saneamento básico, a recreação e o lazer (Zhang et al., 2010). Embora a maior parte da superfície terrestre seja ocupada por água, somente cerca de $2,5 \%$ são de água doce, com um mínimo de $0,06 \%$ correspondente à água dos rios, lagos, biomassa entre outros. O restante da água doce está no subsolo e nas calotas polares sendo estas de difícil acesso (Esteves, 2011).

A água superficial, subterrânea ou mineral, não deve ser vista como uma mercadoria ou commodity, mas como um recurso hídrico de domínio público e gerido pelas instituições da sociedade, visando não apenas ao objetivo econômico, mas também, o social e o ambiental (Fernandez Júnior, Reydon \& Portugal, 2015).

Os principais indicadores de qualidade da água são os aspectos físicos, químicos e biológicos. Os teores máximos de impurezas permitidos na água são estabelecidos em função dos seus usos. Esses teores constituem os padrões de qualidade, que são fixados por entidades públicas com o objetivo de garantir que a água a ser utilizada para um determinado fim não contenha impurezas que venham a prejudicar à saúde (Costa et. al., 2016).

Dessa forma, a indústria deve possuir um sistema de controle de qualidade que possa garantir que durante o processo produtivo da água mineral natural não ocorra contaminação e possa garantir a qualidade e segurança do produto final, a fim de atender à legislação vigente e às Boas Práticas de Fabricação (Gomes, Santana \& De Carvalho, 2015).

Os legisladores e reguladores devem reconhecer os benefícios potenciais da água engarrafada no fornecimento de água mais segura para consumo dentro e fora de casa, especialmente para aqueles que, de alguma forma, dificilmente terão acesso a um abastecimento de água seguro e confiável em um futuro próximo. Para melhorar a qualidade dos produtos de água envasadas as estruturas regulatórias e de monitoramento devem ser integrados (Williams et al., 2015).

A importância da água não está relacionada apenas as funções que ela apresenta na natureza, mas ao seu papel na saúde, economia e qualidade de vida humana. Do ponto de vista cultural, a água também exerce papel importante fazendo parte da construção e crescimento de civilizações ao longo dos séculos, como a exemplo das civilizações mesopotâmicas e egípcias que se desenvolveram ao longo dos rios Tigre e Eufrates e rio Nilo, respectivamente. De acordo com a Organização 
Mundial de Saúde (OMS), cerca de 780 milhões de pessoas no mundo têm acesso a fontes de abastecimento potencialmente nocivas (Souza et al, 2013).

A contaminação hídrica é uma questão ambiental que está sempre voga, visto que favorece a disseminação de doenças e tem um impacto negativo a população em geral, com a notificação ou não de surtos entéricos (Moraes, 2016). Segundo Machado et al (2021), que realizou uma revisão sobre a caraterização, consumo e qualidade microbiológica da água mineral comercializada no Brasil, foi possível verificar que a maioria dos artigos analisados descrevia a presença de pelo menos um microrganismo patogênico, como: Coliformes totais, Escherichia coli (E.coli), Pseudomonas (P. aeruginosa) e Clostridium perfringens (C. perfinges), sendo que em alguns locais mais da metade das águas vendidas nos comércios estavam fora do padrão estabelecido.

Portanto, a água mineral natural é considera de boa qualidade quando obedece às condições higiênico-sanitárias e as boas praticas de fabricação (BPL) durante a sua captação, processamento e envasamento, garantindo ausência de risco à saúde do consumidor (Resende \& Prado, 2008). Sabendo dá importância da água mineral para o consumo humano, o presente trabalho teve como objetivo analisar os padrões da qualidade das águas minerais comercializadas em galões de $20 \mathrm{~L}$, no município de Belém-PA, verificar sua conformidade com relação à legislação vigente e comparar com outros autores.

\section{Metodologia}

A natureza da pesquisa foi aplicada com abordagem mista, pois apresentou características qualitativas e quantitativas (Pereira et al, 2018). Foram coletadas sete marcas, sendo que de seis marcas foram coletas duas amostras de cada, uma no ano de 2020 e outra em 2021; e da sétima marca foram necessárias três coletas, duas no ano de 2020 e uma no ano de 2021, pois foram realizadas denúncias sobre a mesma, totalizando 15 amostras. As amostras foram coletadas em suas embalagens originais lacradas por agentes da vigilância sanitária, no período de janeiro de 2020 a março de 2021, que envolveu os municípios de Ananindeua, Benevides, Castanhal, Santa Barbara e Santa Izabel, onde estão localizadas as indústrias de água mineral (Figura 1). Posteriormente, foram encaminhadas ao Laboratório Central do Pará para a realização das análises dos parâmetros físico-químicos ( $\mathrm{pH}$, condutividade, cloro residual livre, ferro, nitrato, nitrito e cloreto) e dos microbiológicos (Coliformes totais, E.Coli, Pseudonomas Aeruginosa, Enterococcus e Clostridium sulfito redutor)

Figura 1 - Área de Coleta de Amostras.

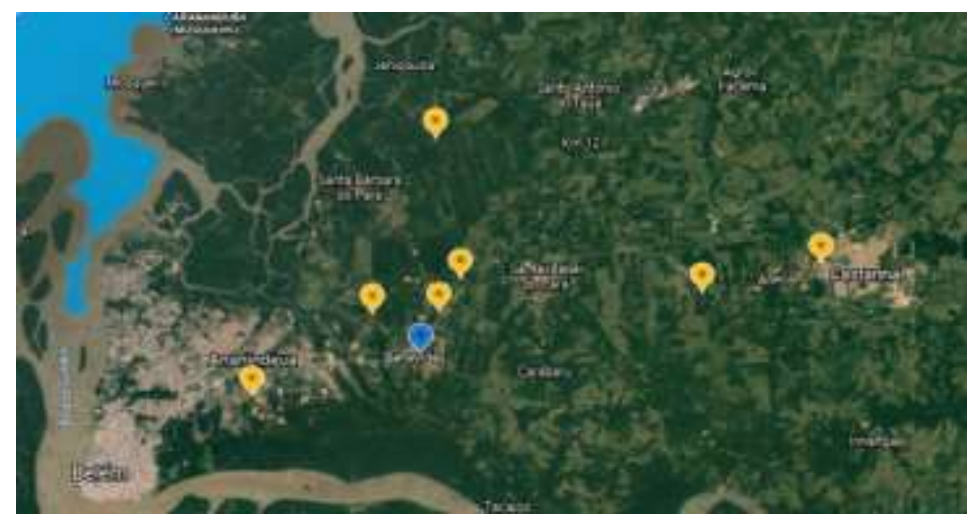

Fonte: Google Maps (2021).

As metodologias analíticas aplicadas nos ensaios práticos são consolidadas e descritas nos Procedimentos Operacionais Padrões (POPs) dos setores de Microbiologia e de Físico-química da Unidade Laboratorial. Os POPs são 
baseados no Standard Methods for Examination of Water and Waste Water (Apha, 2012) e Métodos Físico-Químicos para Análises de Alimentos (IAL, 2008)

\section{Análise dos Parâmetros Físico-químicos}

$p H$ : foi mensurado pelo método da potenciometria usando um Medidor de $\mathrm{pH}$ de bancada digital com compensação manual de temperatura da marca Analion modelo:PM608.

Condutividade: foi realizada pelo método da condutivimetria usando o medidor de condutividade elétrica Orion Star

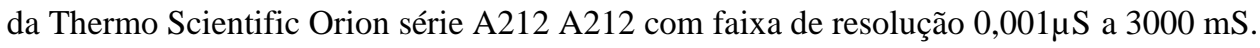

Cloro residual livre, ferro, nitrato, nitrito: foi realizada por reação colorimétrica e com leituras da intensidade de cor realizada no espectrofotômetro da HACH DR 3900.

Cloreto: foi verificado pelo método titulação de precipitação usando como indicador o cromato de potássio a $25 \%$ e como solução titulante o nitrato de prata a $0,014 \mathrm{~N}$.

\section{Análise dos Parâmetros Microbiológico}

Coliformes Totais, E. Coli: foi determinado pelo método enzima substrato cromogênico usando o reagente Collilert. A identificação da presença de $E$. coli foi realizada usando luz ultravioleta.

Pseudonomas Aeruginosa técnica de membrana filtrante (M.F): foi realizada pela técnica de membrana filtrante (M.F) o método baseia-se na filtração de volumes adequados da água a serem analisadas, através de membrana filtrante com porosidade de $0,45 \mu \mathrm{m}$. As bactérias a serem detectadas apresentam dimensões maiores, que ficarão retidas na superfície da membrana a qual foi então transferida para o meio nutritivo e diferencial (meio ágar centrimide) incubar a $35^{\circ} \mathrm{C} \pm 2 \% / 24$ a $48 \mathrm{~h} \pm 2 \mathrm{~h}$. Após incubação no ágar centrimide, observar se houver a produção de um pigmento esverdeado, fluorescente sob a luz ultravioleta.

Enterococcus pela Técnica dos Tubos Múltiplos (T.M): foi realizada pela Técnica dos Tubos Múltiplos (T.M) esse metódo consiste na semeadura de $10 \mathrm{ml}$ da amostra (água) em $10 \mathrm{ml}$ de caldo triptona de soja (TSB) duplo em série de 10 tubos, que foram incubados a $35^{\circ} \mathrm{C} \pm 2$, durante $24 \mathrm{~h}-48 \mathrm{~h}$. A turvação ou formação de precipitado no meio é resultado presuntivo para Enterococcus neste ensaio foi acompanhado por um tubo contendo a cepa ATCC de Enterococcus faecalis (ATCC 29213). Dos tubos positivos, foi retirada uma alçada e então inoculada em caldo Etilvioleta azida (EVA), e incubados a $35^{\circ} \mathrm{C} \pm 2{ }^{\circ} \mathrm{C}$, por 24-48h. Dos tubos de caldo EVA com resultado positivo, foi transferido uma alçada para as placas de Agar Bile-esculina, correspondente a cada tubo positivo; foi incubada a placa em posição invertida durante $24 \mathrm{~h}$ a $35^{\circ} \mathrm{C} \pm 2^{\circ} \mathrm{C}$; Após o período de incubação determinada, foi efetuada a leitura, considerando as colônias castanhas enegrecida, com halo marrom; Das colônias castanho enegrecidas com halo marrom, foi semeada em caldo Brain Heart Infusiom (BHI) sem $\mathrm{NaCl}$ incubadas a $45^{\circ} \mathrm{C} \pm 2 / 48 \mathrm{~h}$ e em caldo $\mathrm{BHI}$ com $\mathrm{NaCl}$ a $6,5 \%$ e incubar a $35^{\circ} \mathrm{C} \pm 2 / 48 \mathrm{~h}$; Com os dados obtidos, foi calculado Número Mais Provável $/ \mathrm{mL}(\mathrm{NMP} / \mathrm{mL})$ de Enterococcus.

Clostridium Sulfito Redutor pela Técnica dos Tubos Múltiplos (T.M): foi realizada pela Técnica dos Tubos Múltiplos (T.M) esse método consiste em aquecer as amostras em banho-maria a $75^{\circ} \mathrm{C}$ durante 10 minutos para a eliminação de organismos não esporulados e formas vegetativas. Foi aquecida em água fervente durante 10 minutos os tubos de caldo diferencial enriquecido para Clostridium (DRCM) concentração dupla a serem utilizados para remover o ar existente neles e esfriá-los rapidamente em água fria (choque térmico); foi preparada uma solução com 50\% de citrato férrico a 7\% e 50\% de sulfito de sódio a 4\% e adicionada $0,4 \mathrm{ml}$ desta solução aos tubos de DRCM; foi inoculada $10 \mathrm{ml}$ da amostra já aquecida em 10 tubos de DRCM, lentamente na parede do tubo para evitar aeração e oxigenação; foi incubado os tubos em jarra de anaerobiose juntamente com o sachê de geração de anaerobiose a $35^{\circ} \mathrm{C} \pm 2$ durante 48 horas; Após o período de incubação 
determinada, foi efetuada a leitura, considerando como positivo os tubos que apresentarem enegrecimento e/ou turvação do meio. Dentre os positivos foi inoculado $0,1 \mathrm{ml}$ da cultura positiva de cada tubo de DRCM, e transferido para o tubo contendo o meio tornassolado correspondente; foi incubado os tubos em jarra de anaerobiose juntamente com o sachê de geração de anaerobiose a $35^{\circ} \mathrm{C} \pm 2$ durante 48 horas; após a incubação determinada foi realizado a leitura considerando como positivo os tubos que apresentarem formação de coágulos, acidificação (coloração rosa do meio de cultura), formação de gás e rompimento dos coágulos; foi feita a transferência de cada tubo positivo de leite tornassolado para o caldo BHI não fervido (teste de aerobiose) e caldo BHI fervido (teste de anaerobiose) simultaneamente; foi incubada a $35^{\circ} \mathrm{C}\left( \pm 2^{\circ} \mathrm{C}\right)$ durante $24 \mathrm{~h}$ $( \pm 3 \mathrm{~h}$ ); foi feita a Coloração de Gram de cada tubo positivo de leite tornassolado e de caldo BHI fervido; para confirmar se é bacilo gram positivo, sendo então considerado positivo para Clostridium Sulfito Redutor.

Os valores quantitativos foram tabulados e aplicados parâmetros estatísticos descritivos no programa Microsoft Excel (2013).

\section{Resultados e Discussão}

Com base nas análises laboratoriais dos parâmetros microbiológicos das 15 amostras, foi possível verificar que 3(três) amostras tiveram resultados positivos, sendo que em 1 amostra foi detectada a presença de Coliformes totais e Clostrídios e em 2 amostras apenas Coliformes totais, acima do limite tolerado pela Resolução no 275 de 22 de setembro de 2005, resolução referente ao regulamento técnico de características microbiológicas para água mineral natural e água natural, fator este que levou ao enquadramento das amostras como insatisfatórias ou impróprias para o consumo (Brasil, 2005ª).

A Tabela 1 apresenta as 15 amostras, com a identificação de entrada no LACEN, os Valores Máximos Permitidos (VPM) pela legislação, e os resultados laboratoriais microbiológicos em Número Mais Provável (NMP) para Enterococcus e Clostridium Sulfito Redutor, em Unidades Formadoras de Colônias (UFC) para Pseudomonas aeruginosa e ausência ou presença de Coliformes totais e Escherichia coli.

Tabela 1 - Resultados Laboratoriais dos ensaios microbiológicos das amostras de Água Mineral.

\begin{tabular}{|c|c|c|c|c|c|}
\hline \multicolumn{6}{|c|}{ Resultados dos ensaios Microbiológicos } \\
\hline \multirow{2}{*}{ Amostras } & Enterococos & $\begin{array}{c}\text { Clostrídios } \\
\text { sulfito redutores }\end{array}$ & \multirow{2}{*}{$\begin{array}{c}\begin{array}{c}\text { Pseudomonas } \\
\text { aeruginosa }\end{array} \\
\mathrm{VMP}(<1,0 \\
\mathrm{UFC} / 100 \mathrm{~mL})^{*}\end{array}$} & Coliformes Totais & Escherichia coli \\
\hline & \multicolumn{2}{|c|}{$\operatorname{VMP}(<1,1 \mathrm{NPM} / 100 \mathrm{~mL})^{*}$} & & \multicolumn{2}{|c|}{ Ausência em 100 mL* } \\
\hline $054 / 2020$ & $\begin{array}{c}<1,1 \mathrm{NPM} / 100 \\
\mathrm{~mL}\end{array}$ & $\begin{array}{l}<1,1 \mathrm{NMP} / 100 \\
\mathrm{~mL}\end{array}$ & $<1,0 \mathrm{UFC} / 100 \mathrm{~mL}$ & $\begin{array}{l}\text { Presença em } 100 \\
\text { mL }\end{array}$ & $\begin{array}{l}\text { Ausência em } \\
100 \mathrm{~mL}\end{array}$ \\
\hline $331 / 2020$ & $\begin{array}{c}<1,1 \mathrm{NPM} / 100 \\
\mathrm{~mL}\end{array}$ & $\begin{array}{c}<1,1 \mathrm{NMP} / 100 \\
\mathrm{~mL}\end{array}$ & $<1,0 \mathrm{UFC} / 100 \mathrm{~mL}$ & $\begin{array}{l}\text { Ausência em } 100 \\
\mathrm{~mL}\end{array}$ & $\begin{array}{l}\text { Ausência em } \\
100 \mathrm{~mL}\end{array}$ \\
\hline $342 / 2020$ & $\begin{array}{c}<1,1 \mathrm{NPM} / 100 \\
\mathrm{~mL}\end{array}$ & $\begin{array}{l}<1,1 \mathrm{NMP} / 100 \\
\mathrm{~mL}\end{array}$ & $<1,0 \mathrm{UFC} / 100 \mathrm{~mL}$ & $\begin{array}{l}\text { Ausência em } 100 \\
\text { mL }\end{array}$ & $\begin{array}{l}\text { Ausência em } \\
100 \mathrm{~mL}\end{array}$ \\
\hline $343 / 2020$ & $\begin{array}{c}<1,1 \mathrm{NPM} / 100 \\
\mathrm{~mL}\end{array}$ & $\begin{array}{c}<1,1 \mathrm{NMP} / 100 \\
\mathrm{~mL}\end{array}$ & $<1,0 \mathrm{UFC} / 100 \mathrm{~mL}$ & $\begin{array}{l}\text { Presença em } 100 \\
\text { mL }\end{array}$ & $\begin{array}{l}\text { Ausência em } \\
100 \mathrm{~mL}\end{array}$ \\
\hline $344 / 2020$ & $\begin{array}{c}<1,1 \mathrm{NMP} / 100 \\
\mathrm{~mL}\end{array}$ & $\begin{array}{c}<1,1 \mathrm{NMP} / 100 \\
\mathrm{~mL}\end{array}$ & $<1,0 \mathrm{UFC} / 100 \mathrm{~mL}$ & $\begin{array}{c}\text { Ausência em } 100 \\
\text { mL }\end{array}$ & $\begin{array}{c}\text { Ausência em } \\
100 \mathrm{~mL}\end{array}$ \\
\hline $345 / 2020$ & $\begin{array}{c}<1,1 \mathrm{NMP} / 100 \\
\mathrm{~mL}\end{array}$ & $\begin{array}{c}<1,1 \mathrm{NMP} / 100 \\
\mathrm{~mL}\end{array}$ & $<1,0 \mathrm{UFC} / 100 \mathrm{~mL}$ & $\begin{array}{c}\text { Ausência em } 100 \\
\mathrm{~mL}\end{array}$ & $\begin{array}{l}\text { Ausência em } \\
100 \mathrm{~mL}\end{array}$ \\
\hline $353 / 2020$ & $\begin{array}{c}<1,1 \text { NMP } / 100 \\
\mathrm{~mL}\end{array}$ & $\begin{array}{c}<1,1 \mathrm{NMP} / 100 \\
\mathrm{~mL}\end{array}$ & $<1,0 \mathrm{UFC} / 100 \mathrm{~mL}$ & $\begin{array}{l}\text { Ausência em } 100 \\
\text { mL }\end{array}$ & $\begin{array}{c}\text { Ausência em } \\
100 \mathrm{~mL} \\
\end{array}$ \\
\hline $498 / 2020$ & $\begin{array}{l}<1,1 \mathrm{NMP} / 100 \\
\mathrm{~mL}\end{array}$ & $\begin{array}{l}<1,1 \mathrm{NMP} / 100 \\
\mathrm{~mL}\end{array}$ & $<1,0 \mathrm{UFC} / 100 \mathrm{~mL}$ & $\begin{array}{l}\text { Ausência em } 100 \\
\text { mL }\end{array}$ & $\begin{array}{l}\text { Ausência em } \\
100 \mathrm{~mL}\end{array}$ \\
\hline $701 / 2020$ & $\begin{array}{c}<1,1 \mathrm{NMP} / 100 \\
\mathrm{~mL}\end{array}$ & $\begin{array}{l}<1,1 \mathrm{NMP} / 100 \\
\mathrm{~mL}\end{array}$ & $<1,0 \mathrm{UFC} / 100 \mathrm{~mL}$ & $\begin{array}{l}\text { Ausência em } 100 \\
\text { mL }\end{array}$ & $\begin{array}{c}\text { Ausência em } \\
100 \mathrm{~mL}\end{array}$ \\
\hline $129 / 2021$ & $<1,1 \mathrm{NMP} / 100$ & $<1,1 \mathrm{NMP} / 100$ & $<1,0 \mathrm{UFC} / 100 \mathrm{~mL}$ & Ausência em 100 & Ausência em \\
\hline
\end{tabular}




\begin{tabular}{c|c|c|c|c|c}
\hline & $\mathrm{mL}$ & $\mathrm{mL}$ & & $\mathrm{mL}$ & $100 \mathrm{~mL}$ \\
\hline $131 / 2021$ & $\begin{array}{c}1,1 \mathrm{NMP} / 100 \\
\mathrm{~mL}\end{array}$ & $\begin{array}{c}<1,1 \mathrm{NMP} / 100 \\
\mathrm{~mL}\end{array}$ & $<1,0 \mathrm{UFC} / 100 \mathrm{~mL}$ & $\begin{array}{c}\text { Ausência em } 100 \\
\mathrm{~mL}\end{array}$ & $\begin{array}{c}\text { Ausência em } \\
100 \mathrm{~mL}\end{array}$ \\
\hline $132 / 2021$ & $\begin{array}{c}<1,1 \mathrm{NMP} / 100 \\
\mathrm{~mL}\end{array}$ & $\begin{array}{c}<1,1 \mathrm{NMP} / 100 \\
\mathrm{~mL}\end{array}$ & $<1,0 \mathrm{UFC} / 100 \mathrm{~mL}$ & $\begin{array}{c}\text { Ausência em } 100 \\
\mathrm{~mL}\end{array}$ & $\begin{array}{c}\text { Ausência em } \\
100 \mathrm{~mL}\end{array}$ \\
\hline $142 / 2021$ & $\begin{array}{c}<, 1 \mathrm{NMP} / 100 \\
\mathrm{~mL}\end{array}$ & $\begin{array}{c}<1,1 \mathrm{NMP} / 100 \\
\mathrm{~mL}\end{array}$ & $<1,0 \mathrm{UFC} / 100 \mathrm{~mL}$ & $\begin{array}{c}\text { Ausência em } 100 \\
\mathrm{~mL}\end{array}$ & $\begin{array}{c}\text { Ausência em } \\
100 \mathrm{~mL}\end{array}$ \\
\hline $152 / 2021$ & $\begin{array}{c}<1,1 \mathrm{NMP} / 100 \\
\mathrm{~mL}\end{array}$ & $\begin{array}{c}<1,1 \mathrm{NMP} / 100 \\
\mathrm{~mL}\end{array}$ & $<1,0 \mathrm{UFC} / 100 \mathrm{~mL}$ & $\begin{array}{c}\text { Ausência em } 100 \\
\mathrm{~mL}\end{array}$ & $\begin{array}{c}\text { Ausência em } \\
100 \mathrm{~mL}\end{array}$ \\
\hline $153 / 2021$ & $\begin{array}{c}<1,0 \mathrm{UFC} / 100 \\
\mathrm{~mL}\end{array}$ & 2,2 \\
& $\mathrm{NMP} / 100 \mathrm{~mL}$ & $<1,0 \mathrm{UFC} / 100 \mathrm{~mL}$ & $\begin{array}{c}\text { Presença em } 100 \\
\mathrm{~mL}\end{array}$ & $\begin{array}{c}\text { Ausência em } \\
100 \mathrm{~mL}\end{array}$ \\
\hline
\end{tabular}

* Limites toleráveis pela Resolução n 275 de 22 de setembro de 2005

Fonte: HARPYA /DATASSUS, LACEN-PA.

Já os parâmetros Físico - Químicos, as 15 amostras analisadas estavam de acordo com a Resolução nº 274, de 22 de setembro de 2005, resolução referente ao regulamento técnico para águas envasadas e gelo (Brasil, 2005 ${ }^{\text {b }}$. A Tabela 2 apresenta as 15 amostras, com a identificação de entrada no LACEN, os resultados laboratoriais das análises físico-químicos e os valores estabelecidos pela legislação em Limite Máximo Permitido (LMP) para nitrato, nitrito e clore livre, e Valor Máximo Permitido (VMP) para cloreto e ferro.

Tabela 2 - Resultados Laboratoriais dos ensaios Físico-químicos das amostras de Água Mineral.

\begin{tabular}{l|c|c|c|c|c|c|c}
\hline Amostras & $\mathrm{pH}$ & Condutividade & Nitrato & Nitrito & Cloreto & $\begin{array}{l}\text { Cloro } \\
\text { Livre }\end{array}$ & Ferro \\
\hline & \multicolumn{2}{|c|}{ Em conformidade com o rótulo. } & $\begin{array}{c}\text { LMP 50,0 } \\
\mathrm{mg} / \mathrm{L}^{*}\end{array}$ & $\begin{array}{c}\text { LMP 0,02 } \\
\mathrm{mg} / \mathrm{L}^{*}\end{array}$ & $\begin{array}{c}\text { VMP 250,0 } \\
\mathrm{mg} / \mathrm{L}\end{array}$ & $\begin{array}{c}\text { LMP 5 } \\
\mathrm{mg} / \mathrm{L}^{*}\end{array}$ & $\begin{array}{c}\text { VMP 0,3 } \\
\mathrm{mg} / \mathrm{L}^{*}\end{array}$ \\
\hline $054 / 2020$ & - & - & - & - & - & - & - \\
\hline $331 / 2020$ & 4,58 & $32,09 \mu \mathrm{S} / \mathrm{cm}$ & $0,7 \mathrm{mg} / \mathrm{L}$ & $0,007 \mathrm{mg} / \mathrm{L}$ & $11,2 \mathrm{mg} / \mathrm{L}$ & & $0,06 \mathrm{mg} / \mathrm{L}$ \\
\hline $342 / 2020$ & 4,66 & $33,50 \mu \mathrm{S} / \mathrm{cm}$ & $2,4 \mathrm{mg} / \mathrm{L}$ & $0,008 \mathrm{mg} / \mathrm{L}$ & $28,0 \mathrm{mg} / \mathrm{L}$ & $0,01 \mathrm{mg} / \mathrm{L}$ & $0,12 \mathrm{mg} / \mathrm{L}$ \\
\hline $343 / 2020$ & 4,52 & $28,01 \mu \mathrm{S} / \mathrm{cm}$ & $1,8 \mathrm{mg} / \mathrm{L}$ & $0,009 \mathrm{mg} / \mathrm{L}$ & $11,0 \mathrm{mg} / \mathrm{L}$ & $0,05 \mathrm{mg} / \mathrm{L}$ & $0,11 \mathrm{mg} / \mathrm{L}$ \\
\hline $344 / 2020$ & 4,90 & $30,97 \mu \mathrm{S} / \mathrm{cm}$ & $2,3 \mathrm{mg} / \mathrm{L}$ & $0,011 \mathrm{mg} / \mathrm{L}$ & $27,0 \mathrm{mg} / \mathrm{L}$ & $0,03 \mathrm{mg} / \mathrm{L}$ & $0,03 \mathrm{mg} / \mathrm{L}$ \\
\hline $345 / 2020$ & 4,28 & $32,41 \mu \mathrm{S} / \mathrm{cm}$ & $0,8 \mathrm{mg} / \mathrm{L}$ & $0,012 \mathrm{mg} / \mathrm{L}$ & $31,0 \mathrm{mg} / \mathrm{L}$ & $5,0 \mathrm{mg} / \mathrm{L}$ & $0,04 \mathrm{mg} / \mathrm{L}$ \\
\hline $353 / 2020$ & 4,49 & $76,52 \mu \mathrm{S} / \mathrm{cm}$ & $2,7 \mathrm{mg} / \mathrm{L}$ & $0,012 \mathrm{mg} / \mathrm{L}$ & $38,0 \mathrm{mg} / \mathrm{L}$ & $0,01 \mathrm{mg} / \mathrm{L}$ & $0,12 \mathrm{mg} / \mathrm{L}$ \\
\hline $498 / 2020$ & 4,74 & $30,5 \mu \mathrm{S} / \mathrm{cm}$ & $0,7 \mathrm{mg} / \mathrm{L}$ & $0,02 \mathrm{mg} / \mathrm{L}$ & $25,0 \mathrm{mg} / \mathrm{L}$ & $0,02 \mathrm{mg} / \mathrm{L}$ & $0,08 \mathrm{mg} / \mathrm{L}$ \\
\hline $701 / 2020$ & 4,5 & $21,8 \mu \mathrm{S} / \mathrm{cm}$ & - & - & $10,0 \mathrm{mg} / \mathrm{L}$ & $0,01 \mathrm{mg} / \mathrm{L}$ & $0,02 \mathrm{mg} / \mathrm{L}$ \\
\hline $129 / 2021$ & 4,62 & $80,13 \mu \mathrm{S} / \mathrm{cm}$ & - & - & $21,0 \mathrm{mg} / \mathrm{L}$ & $0,01 \mathrm{mg} / \mathrm{L}$ & $0,02 \mathrm{mg} / \mathrm{L}$ \\
\hline $131 / 2021$ & 5,05 & $31,67 \mu \mathrm{S} / \mathrm{cm}$ & - & - & $23,0 \mathrm{mg} / \mathrm{L}$ & $0,02 \mathrm{mg} / \mathrm{L}$ & $0,02 \mathrm{mg} / \mathrm{L}$ \\
\hline $132 / 2021$ & 5,06 & $29,72 \mu \mathrm{S} / \mathrm{cm}$ & - & - & $25,0 \mathrm{mg} / \mathrm{L}$ & $0,01 \mathrm{mg} / \mathrm{L}$ & $0,02 \mathrm{mg} / \mathrm{L}$ \\
\hline $142 / 2021$ & 5,08 & $18,03 \mu \mathrm{S} / \mathrm{cm}$ & - & - & $19,0 \mathrm{mg} / \mathrm{L}$ & $0,02 \mathrm{mg} / \mathrm{L}$ & $0,07 \mathrm{mg} / \mathrm{L}$ \\
\hline $152 / 2021$ & 5,08 & $13,55 \mu \mathrm{S} / \mathrm{cm}$ & - & - & $14,0 \mathrm{mg} / \mathrm{L}$ & $0,01 \mathrm{mg} / \mathrm{L}$ & $<\mathrm{LD}$ \\
\hline $153 / 2021$ & 5,65 & $12,57 \mu \mathrm{S} / \mathrm{cm}$ & - & - & $17,0 \mathrm{mg} / \mathrm{L}$ & $<\mathrm{LD}$ & $0,01 \mathrm{mg} / \mathrm{L}$ \\
\hline
\end{tabular}

* Limites da Resolução n 274, de 22 de setembro de 2005

** < LD: Abaixo do Limite de Detecção

Fonte: HARPYA/ DATASSUS, LACEN-PA.

Á água mineral consumida na região metropolitana de Belém, capital do Estado do Pará, tem características semelhantes às descridas no Decreto-Lei $\mathrm{n}^{\circ}$ 7.841, de 8 de agosto de 1945, que dentre as disposições, diz que são aquelas provenientes de fontes naturais ou de fontes artificialmente captadas que possuem composição química ou propriedades físicas ou físico-químicas distintas das águas comuns, sua composição normal provenientes de fontes naturais ou de fontes artificialmente captadas que preencham tão somente as condições de potabilidade para a região (Brasil, 1945) Logo, a água 
por sua condição natural deve ser considerada como um recurso hídrico de domínio público, onde sua utilização, comercialização e utilização em geral leve em consideração o acesso da população, a sustentabilidade e fundamentalmente a preservação do meio ambiente (Fernandez Júnior, Reydon \& Portugal, 2015).

Os resultados das análises microbiológicas mostraram a ocorrência de coliformes totais em amostras coletadas no ano de 2020 e 2021, que conforme dados do laboratório existe reincidência de um mesmo fabricante, fato este relevante pois as doenças transmitidas por águas maculadas por agentes patológicos representam uma importante parcela dos problemas de saúde pública, levando milhares de pessoas a internações hospitalares todos os anos no Brasil (Sant'ana et al, 2003)

As amostras microbiológicas enquadradas como insatisfatórias, caso sejam ingeridas podem causar infecções intestinais e os coliformes, as patologias como: meningites, intoxicações alimentares, infecções urinárias e pneumonias, inclusive as nosocomiais, as infecções causadas por esses organismos são complexas e envolvem múltiplos modos de transmissão (Koneman et al,2018)

$\mathrm{Na}$ avaliação de Coliformes totais e Coliformes fecais/E. coli, Pseudomonas aeruginosa e bactérias heterotróficas, em amostras de água mineral natural, não carbonatada, em garrafões de 20 litros comercializadas nas cidades de Araraquara e Américo Brasiliense - SP, das 21 marcas de água mineral analisadas verificou que 13 amostras de 8 marcas apresentaram positividade para coliformes totais. Cabe ressaltar que os resultados obtidos nessa pesquisa também mostraram essa tendência de contaminação das amostras por coliformes totais, uma vez que em três amostras analisadas foram encontrados Coliformes totais acima do limite especificado pela Resolução nº 275, de 22 de Setembro de 2005. (Farache Filho \& Dias, 2008)

$\mathrm{Na}$ avaliação microbiológica das amostras de águas minerais comercializadas em garrafões de $20 \mathrm{~L}$ na região metropolitana de Recife, Estado de Pernambuco, também concluíram que houve contaminação microbiológica em parte das amostras analisadas, e que por isso não atenderam aos parâmetros estabelecidos pela legislação em vigor. Sendo que essas contaminações microbiológicas podem ocorrer pela falta de boas práticas ou devido ao reaproveitamento dos garrafões de $20 \mathrm{~L}$ pelas indústrias de envasam água mineral, visto que esta forma de comercialização vem apresentando maior índice de contaminação por falhas na higiene dos recipientes (Coelho et al, 2010).

Os resultados de Silveira Neta et al (2012) sobre qualidade físico-química e microbiológica de cinco marcas de águas minerais comercializadas em Belém, no Estado do Pará, se mostraram impróprias para o consumo humano de acordo com as análises efetuadas, o que demonstra a importância da de Boas Práticas de Fabricação nas empresas que realizam o envase deste tipo de produto.

Gomes, Santana e De Carvalho (2015) analisaram galões de 20L de água no município de Itabuna, na Bahia, e constataram a contaminação por enterobactérias, isso ocorre em virtude de a água mineral ser um produto não estéril e que pode ser contaminada por micro-organismos caso ocorram falhas higiênico-sanitárias durante o transporte e/ou armazenamento.

No estudo de Vinhal, Brasil e Moura (2018) foi constatado que a água mineral engarrafada consumida pela comunidade da Universidade Federal de Goiás apresentou contagem de microrganismos heterotróficos superior ao limite estabelecido, a presença de microrganismos patogênicos evidencia a necessidade de um controle rigoroso pela Universidade.

Nas análises microbiológicas de águas minerais comercializadas em garrafões de 20 litros, na cidade de Picos-PI também foi possível constatar falhas higiênico-sanitárias em três marcas de água mineral analisadas no primeiro lote foram consideradas impróprias para o consumo por apresentarem contagem superior em comparação com a legislação vigente, já no segundo lote duas marcas apresentaram Coliformes totais acima do valor máximo permitido e resultado positivo para Coliformes termotolerantes (Ribeiro, Barros \& Barros, 2021)

A contaminação por clostrídios sulfitos redutores por sua vez é preocupante nas águas minerais, pois apresentam elevada resistência às condições ambientais adversas. São encontrados no solo e intestino humano e de outros animais, são 
indicadores de condições de contaminação fecal remota. Podem também indicar contaminação por solo do reservatório ou fonte contaminada com águas superficiais, além de falhas nas boas práticas de fabricação (Franco \& Landgraf, 2016).

A RDC n ${ }^{\circ} 173$, estabelece normas para garantir uma melhor qualidade no produto final, está resolução, define as boas práticas de fabricação e como deve ser os procedimentos durante o processo de envase, desde a captação e armazenagem da água, a fabricação e higienização da embalagem, envase e fechamento, a rotulagem o transporte e a exposição à venda. As águas minerais devem permanecer em local seco e ventilado, protegidas contra a luz solar direta, com a temperatura adequada para que não ocorra alterações na qualidade microbiológica e físico-química, não devem ser armazenadas próximas a produtos tóxicos, voláteis, evitando a possível contaminação e impregnação de odores (Brasil, 2006). É fundamental que a indústria possua um rigoroso programa de controle de qualidade durante seu processo produtivo, para que de fato oferte uma água mineral de qualidade a população consumidora (Gomes et al, 2011)

Os valores médios de pH, entre 4,28 e 5,08, sugerem que as águas não são significativamente mineralizadas ou mineralizas artificialmente, quanto aos demais parâmetros físico-químicos analisados as amostras estavam de acordo com o que determina a Resolução RDC 274/2005 ANVISA-MS (Brasil, 2005 ). Cabe ressaltar que os valores de pH estavam de acordo com o declarado no rótulo pelos fabricantes, porém o valor de nitrato, cujo limite estabelecido é de até $50 \mathrm{mg} / \mathrm{L}$, apresentou diferença entre a informação do rótulo e os resultados obtidos no laboratório.

A maioria dos estudos que constam sobre controle de qualidade de água mineral, aponta aquelas comercializadas em garrafões plásticos de 20 litros são as mais propensas à contaminação microbiológica e discordância dos valores das propriedades físico-químicas trazidos em seus rótulos apontam para necessidade de mais estudos de controle de qualidade destas águas para consumo (Reis, Bevilacqua \& Carmo, 2014).

\section{Conclusão}

A água mineral consumida na região metropolitana de Belém, em sua maioria está de acordo com os padrões estabelecidos, visto que o resultado das análises físico-químicas estava em conformidade com a legislação e em relação as análises microbiológicas três amostras apresentaram contaminação por Coliformes totais e Clostrídios.

A presença de microrganismos indica a necessidade de estudos rotineiros sobre a qualidade da água mineral comercializada em Belém, para que os órgãos reguladores e controladores das ações de produção, distribuição e comercialização do Estado do Pará possam estabelecer uma estrutura organizacional, com a finalidade de verificar as várias etapas que a água mineral passa até chegar ao consumidor final em consonância com a legislação federal, e assim estabelecer parâmetros rígidos de controles da água, sem que essa possa causar possíveis riscos de doenças ou prejuízos socioeconômicos.

\section{Referências}

American Public Health Association (APHA); American Water Works Association (AWWA); Water Environment Federation (WEF). (2012) Standard Methods for the Examination of Water and Wastewater. (22a ed.), APHA/AWWA/WEF.

Brasil. (2000) Resolução $n^{\circ}$ 54, de 15 de junho de 2000. Dispõe sobre o regulamento técnico para fixação de identidade e qualidade de água mineral natural e água natural. Diário Oficial da República Federativa do Brasil, Brasília, DF, Seção 1

Brasil ${ }^{\mathrm{a}}$.(2005) Agência Nacional de Vigilância Sanitária (ANVISA).Resolução RDC $n^{\circ} 275$ de 22 de setembro de 2005. Aprova o Regulamento Técnico de Características Microbiológicas para Água Mineral Natural e Água Natural. Diário Oficial da União, Brasília. http://bvsms.saude.gov.br/bvs/saudelegis/anvisa/2005/rdc0275_22_09_2005.html

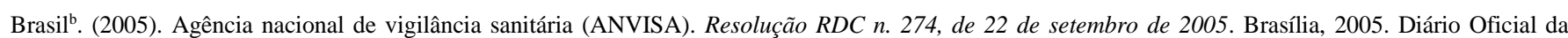
União http://bvsms.saude.gov.br/bvs/saudelegis/anvisa/2005/res0274_22_09_2005.html

Brasil. (2006). Agência Nacional de Vigilância Sanitária. Resolução RDC $n^{\circ} 173$, de 13 de setembro de 2006 . Aprova o Regulamento Técnico de Boas Práticas para Industrialização e Comercialização de Água Mineral Natural e de Água Natural. Brasília, DF http://elegis.anvisa.gov.br/leisref/public/showAct.php?id=23915\&word>. 
Coelho, M. I. S., Mendes, E. S., Cruz, M. C. S., Bezerra, S. S. \& Silva, R. P. P. (2010) Avaliação da qualidade microbiológica de águas minerais consumidas na região metropolitana de Recife, Estado de Pernambuco, Acta Scientiarum. Health Sciences Maringá, 32(1),1-8

Costa, P. S. F., et. al. (2016) Avaliação da qualidade das águas superficiais da sub-bacia do rio Capivari no município de Chapada do Norte - MG. Caderno de Geografia, 26(1), 2318-2962.

De Moraes, P. A. (2016). Perfil epidemiológico e qualidade microbiológica da água para consumo humano envolvida em surtos de doenças de veiculação hídrica ocorridos em minas gerais no período de 2014 a 2016 através da pesquisa de indicadores de contaminação fecal. 58f. Tese (Especialização). Departamento de Microbiologia - Instituto De Ciências Biológicas Universidade Federal De Minas Gerais. 2016

Esteves, F. (2011). Fundamentos de limnologia. (3a ed.), Interciência, 826 p.

Farache Filho, A. \& Dias, M. F. F. (2008) Qualidade Microbiológica de Águas Minerais em Galões de 20 Litros, Alim. Nutr.19 (3), $243-8$.

Fernandez Júnior, P. S. P., Reydon, B. P. \& Portugal, N. S. (2015) As águas minerais no Brasil: uma análise do mercado e da institucionalidade para uma gestão integrada e sustentável. Rev. Ambient. Água. 10(2), 414-30

Franco, B. D. G. M., \& Landgraf, M. (2016) Microbiologia dos alimentos. (2a ed.), Atheneu

Gomes, T. V. D, Silva, M. R., Da Conceição, C. \& Azeredo, D. R. P. (2011) Proposta de plano para Análise de Perigos e Pontos Críticos de Controle (APPCC) para o processo de industrialização da água mineral. Segurança Alimentar e Nutricional, 18 (1), 31-42

Gomes, T. P., Santana, J. S. \& De Carvalho, L. R. (2015) Avaliação microbiológica de água mineral comercializada no município de Itabuna-Bahia. Revista Eletrônica de Farmácia, 12 (4), 65-72.

Instituto Adolfo Lutz (IAL). (2008) Métodos físico-químicos para análise de alimentos/. IV edição São Paulo: Instituto Adolfo Lutz.

Júnior. P. S. P., Reydon. B. P., \& Portugal. N. S. (2015). As águas minerais no Brasil: uma análise do mercado e da institucionalidade para uma gestão integrada e sustentável. Rev. Ambient. Água (10)2, 414-29

Koneman, E. W. et al (2018) Diagnóstico Microbiológico. (7a ed.), MEDSI.

Machado, R. H. L., Marinho, L. F. C., Dworak, E. S., Carolino, I. D. R., Conegero, C. I. \& Massago, M. (2021). Água mineral comercializada no Brasil: caracterização, consumo e qualidade microbiológica. Arquivos do Mudi. 25(1),12 - 25.

Pereira, A. S., Shitsuka, D. M., Parreira, F. J \& Shitsuka, R. (2018). Metodologia da pesquisa científica. UFSM

Ribeiro, T. I., Barros, F. A. A. \& Barros, S. B. A. (2021) Análise microbiológica de águas minerais de garrafões de 20 litros comercializada na cidade de Picos-PI. Brazilian Journal of Development, 7(2), 15487-99

Reis, L. R., Bevilacqua, P. D. \& Carmo, R. F. (2014) Água envasada: qualidade microbiológica e percepção dos consumidores no município de Viçosa (MG), Rio de Janeiro, RJ, Cad. Saúde Colet., 22 (3), 224-32

Resende, A. \& Prado, C. N. (2008). Perfil microbiológico da água mineral comercializada no Distrito Federal. Revista de Saúde e Biologia, 3(2), 16-22

Sant'ana, A. S., Silva, S. C. F. L., Junior Farani, C. H. R \& Macedo, V. F. (2003) Qualidade microbiológica de águas minerais. Campinas, Cienc. Tecnol. Aliment. 23 190-4.

Silveira Neta, J. J. S., Mattietto R. A, Carvalho, A. V., Da Costa, R. F., \& Lima, A. C. C. (2012) Avaliação físico-química e microbiológica de águas minerais comercializadas em Belém-Pará. In: Simpósio De Segurança Alimentar. Retorno Às Origens? Anais. 4 Gramado: SBCTA-RS, 2012.

Souza J. R., De Moraes M. E. M., Sonada S. L. \& Santos, H. C. R. G. (2013) A Importância da Qualidade da Água e os seus Múltiplos Usos: Caso Rio Almada, Sul da Bahia, Brasil. REDE - Revista Eletrônica do Prodema, 8(1), 26-45.

Vinhal, D. C., Brasil, A. L. M. \& Moura, D. B. C. (2018). Comparação microbiológica entre água mineral e água de fonte pública consumidas na Universidade Federal de Goiás. Revista Científica FacMais, 14(3), 30-40

Williams, A. R., Bain, R. E. S., Fisher, M. B., Cronk, R., Kelly, E. R. \& Bartram, J. (2015) Uma revisão sistemática e meta-análise de contaminação fecal e tratamento inadequado de água engarrafada. Plos one. 10(10), e0140899-926. 10.1371/ journal.pone.0140899.

Zhang, Z., Tao, F., Du, J., Shi, P., Yu, D., Meng, Y. et al. (2010). Surface water quality and its control in a river with intensive human impacts-a case study of the Xiangjiang River, China. Journal of Environmental Management, 91(12) 2483-90. http://dx.doi.org/10.1016/j.jenvman.2010.07.002 